\title{
An On-line Monitoring and Flight Inspection System Based on Unmanned Aerial Vehicle for Navigation Equipment
}

\author{
Ping Yang ${ }^{\text {a, }}{ }^{,}$, Jiaquan Ye $^{b}$, Jing Liu ${ }^{c}$, Zhengbo Yang ${ }^{d}$ and Fei Liang ${ }^{e}$ \\ The Second Research Institute of CAAC, Chengdu 610041, China. \\ *a yangping@caacsri.com, ${ }^{\mathrm{b}}$ yejiaquan@caacsri.com, ${ }^{\mathrm{c}}$ liujing@caacsri.com \\ d yangzhengbo@caacsri.com,e liangfei@caacsri.com
}

\begin{abstract}
Keywords: Navigation equipment, Air Traffic Control, ILS, VOR, DME and NDB, ICAO DOC 8071.
\end{abstract}

\begin{abstract}
Navigation equipment is the most important infrastructure of civil aviation, which is closely related to flight safety. In addition to regular flight inspection, navigation equipment's patrol measuring, maintenance measuring, running measuring under special weather conditions are the important means of ensuring aviation flight safety. According to the safety maintenance requirements of Civil Aviation Air Traffic Control navigation equipment, this paper developed one on-line monitoring and flight inspection system with independent intellectual property rights based on unmanned aerial vehicle for navigation equipment, the system breakthroughs the key technologies of measuring navigation equipment on-line including ILS, VOR, DME and NDB, which also meets the requirements of navigation equipment ground measurement set by the ICAO DOC 8071, it provides technical means of the on-line measurement and flight inspection for navigation equipment, improves the safety of navigation equipment operation, and reduces the impact of measuring navigation equipment on airport operation.
\end{abstract}

\section{Introduction}

Civil aviation navigation equipment is very important to ensuring aviation flight safety, measuring the equipment is now highly regarded at home and abroad. International Civil Aviation Organization (ICAO), International Radio Consultative Committee (CCIR) and the International Special Committee on Radio Interference (CISPR) and other international organizations have developed and issued related documents of navigation equipment, that include inspection and maintenance methods of navigation equipment. The Civil Aviation Administration of China (CAAC) also has developed relevant measure procedures, requirements and flight inspection criterion of aviation equipment.

Navigation equipment is the most important infrastructure of civil aviation and is closely related to flight safety. In the past 20 years, with the rapid development of electronic and computer technology, civil navigation equipment testing technology has also been greatly developed. As the indispensable important aviation equipment in the process of developing, producing, testing and maintaining, the Instrument Landing System (ILS, including: Localizer, Glide path and Marker beacon)/VHF Omni-directional Range (VOR)/ Distance Measuring Equipment (DME)/Non-directional Radio Beacon (NDB) test equipment plays an important role in the process of navigation equipment operation, and receives mostly attention from the industry. The number of general airport will be reach to 500 in 2020, with the increasingly number of the new airport, take-offs and landing, the CAT II/III ILS implementing in the large domestic airports, the airport running will be more and more dependent on the navigation equipment, which puts forward higher requirements of the navigation equipment performance and operation security $[1,2]$.

As one of the most basic premise for airport operation, flight inspection is the necessary means to ensures the communications, navigation, radar and other facilities to meet the requirements of the normal operation of flight, it is also an important part of the safety of flight, passengers and property [3]. Flight inspection is divided into four categories: production inspection, monitoring inspection, periodic inspection and special inspection. It is mandatory for the airports to flight inspecting 
according to the ICAO and the CAAC. For example: new airports must be flight inspected before they running, CAT I ILS must be flight inspected once every 270 days, VOR must be flight inspected once every 540 days $[4,5]$. The current domestic and international flight inspection are made by professional pilots with special planes and special inspection equipment. With the increasing number of airport, and new buildings around the airport, the demand of flight inspection is more and more frequent, but currently the pilots of flight inspection are limited, the available planes of flight inspection do not exceed 20 in our country, thus cannot response to the airports in need for flight inspecting in time, so as to impact the operation of the airport [6].

In addition to regular flight inspection, the navigation equipment 's patrol test, maintenance test and operation test under special weather conditions have become the important means to guarantee the safety of aviation flight. According to the ground measurement requirements of the navigation equipment, the Europeans and the United States have been researched on ILS and VOR ground testing tools since 1990s, they also have developed the corresponding exclusive testing tools such as Marconi IFR4000, R\&S EVS300 ILS/VOR analyzer, R\&S ILS Checker software, R\&S TS6300 ILS test system, and general equipment such as spectrum analyzer, oscilloscope, network analyzer. The exclusive testing tools are expensive and lack independent intellectual property rights. The general equipment has the disadvantages of single function and large interference from human factors. At present, the on-line measurement technology of navigation equipment in China is mainly focus on the application of general equipment and researching the measurement methods $[7,8]$.

For the moment, the traditional means of ground test for navigation equipment need to shut down the power, and their testing index are not comprehensive, test result are discontinuity, that cannot fully reflect the running state of navigation equipment, also impact the operation of the airport. The company R\&S has developed a mobile-based measuring technique, which is improved compare to the traditional testing methods on the ground, can measures signal of navigation equipment in the near field in the light of sight condition, but it is limited to roads, terrain, obstacles and measurement height, the far field testing results cannot be reflecting the navigation equipment's running status effectively and reliability, it cannot be used for flight inspecting further.

\section{An On-line Monitoring and Flight Inspection System Based on Unmanned Aerial Vehicle for Navigation Equipment}

This paper provides an on-line monitoring and flight inspection system based on the unmanned aerial vehicle (UAV) for navigation equipment, to improve the precision and accuracy of measurement and flight inspection and reduce their impact on airport operation.

\subsection{The Structure of an On-Line Monitoring and Flight Inspection System}

The on-line monitoring and flight inspection system includes GPS antenna, GPS positioning module, sensor module, airborne computer module, actuator module, on-line monitoring and flight inspection system module, ground control station, as shown in Fig.1. The on-line monitoring and flight inspection system module is consisting of central controller, measurement antenna, RF acquisition and processing module, signal monitoring and analysis module, and flight inspection module.

GPS antenna is used for receiving the satellite signal. GPS positioning module is used for processing satellite signal from GPS antenna, acquiring the positioning information of the UAV during its flying, and then sending the positioning information to airborne computer module and central controller. Sensor module is used for obtaining the flight status information of UAV during its flying, which including speed information, profile information and course information, and then sending this information to airborne computer module. Airborne computer module is used for sending flight orders to the actuator module according to the configured flight path and flight status information. Actuator module is used for executing flight orders to enable the UAV to fly along the configured flight path. Ground control station is used for receiving information from airborne computer module and sending the command information to airborne computer module to adjust flight missions. 


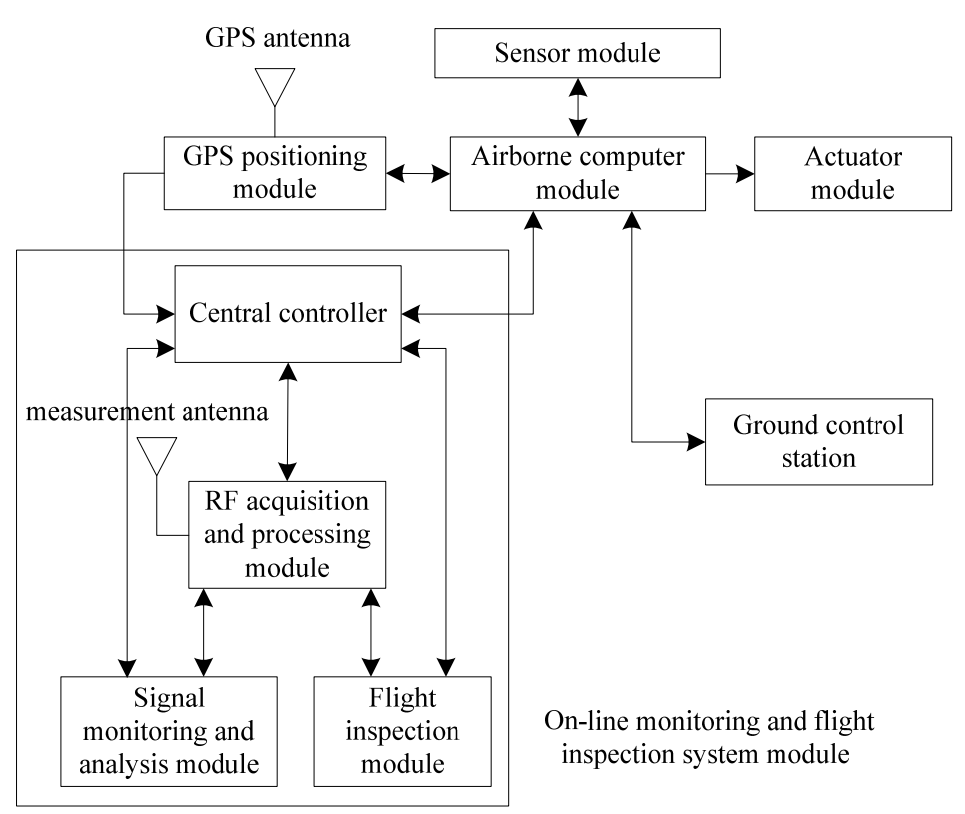

Figure 1. The structure of an on-line monitoring and flight inspection system

In the on-line monitoring and flight inspection system module, measurement antenna is used for receiving the signal of ILS/VOR/DME/NDB. Central controller is used for configuring flight mission, receiving the positioning information and sending to the RF acquisition and processing module, the flight mission including: the navigation equipment and its working frequency to be measured, configured flight path, the characteristic parameters of signals to be analyzed. RF acquisition and processing module is used for acquiring and processing navigation equipment's signal according to the central controller, which has configured to the measuring navigation equipment and its frequency signal, getting the parameters data and the positioning information of each sampling point, then recording and storing the parameters data and positioning information at the current moment's sampling point. Signal monitoring and analysis module obtains parameters data and positioning information from the RF acquisition and processing module, monitoring and analyzing according to the parameters data and positioning information. Flight inspection module obtains the parameters data and positioning information from the RF acquisition and processing module, and then analyzing the flight inspection according to the parameters data and position information.

Like the manned aircraft, the UAV is also able to fixed-point hovering, linear flying, circular flying, ring flying according to the pre-programmed flight path, landing according to the landing route formed by the ILS signal, or flying follow the guidance of the ground control station. It can measure and analyze navigation signals during the flight and transmits measured data to the ground control station in real time. The UAV can also load with a high-definition camera, for recording the ground images during the flight, and drawing three-dimensional (3D) service area signals according to the ground images. It can detect obstacles changing around the airport in time by comparing these 3D service signals regularly and helps the related personnel to evaluate navigation equipment's signal abnormal changes caused by the environmental problems, terrain and buildings, thus offers a rational basis for the corresponding improvement suggestions. 


\subsection{The Theory of an On-Line Monitoring and Flight Inspection System}

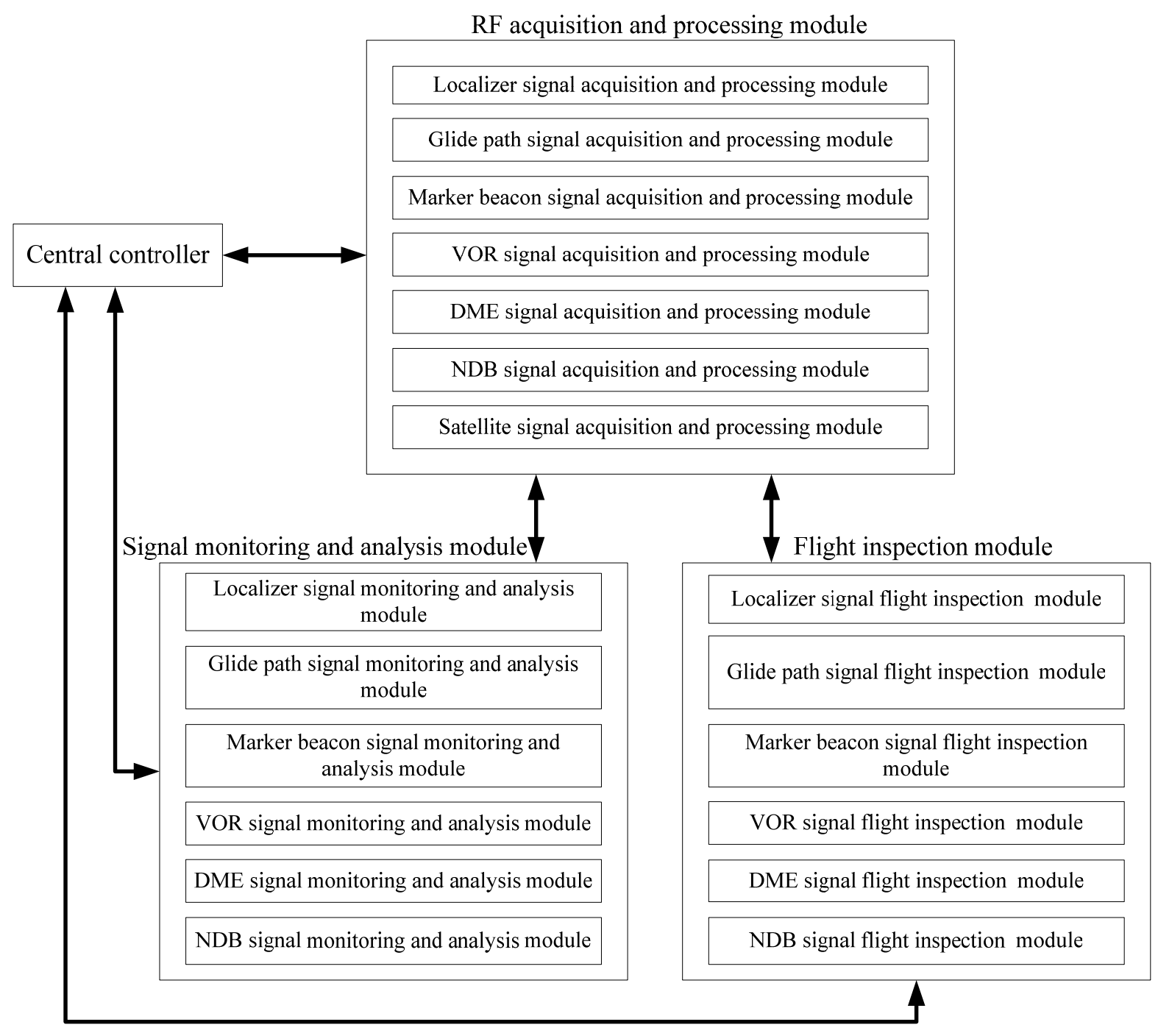

Figure 2. The theory of an on-line monitoring and flight inspection system

As shown in Fig.2, the RF acquisition and processing module includes localizer signal processing module, glide path signal processing module, marker beacon signal processing module, VOR signal processing module, DME signal processing module, NDB signal processing module and satellite signal processing module. Each sub module is independent of each other. In order to reduce the weight of the UAV load and its energy consumption, we can free collocation these modules on the UAV according to our actual requirements. According to the output of the GPS positioning module, the satellite signal acquisition and processing module obtains and records the positioning information (including: the height information, distance and azimuth information) of each sampling point, according to the position information of the measuring navigation equipment located on the runway.

In the signal monitoring and analysis module, each sub module is also independent of each other. According to the allocated monitoring tasks (i.e., determining signal characteristic parameters to be monitored and analyzed), combining with the measured signal at each sampling point, and distance, azimuth and height information of the sample point between the runway entrance or the monitored navigation equipment, every sub module can plot its monitoring diagram, and analyze whether its monitoring results meets the relevant requirements of ICAO DOC8071 or not. For example: localizer signal monitoring and analysis module can plot its monitoring diagram and analyze whether its monitoring results meets the relevant requirement of ICAO DOC8071 or not, according to its allocated monitoring flight mission, combining with the measured signal at each sampling point, the 
distance and height between their location and the runway entrance, and their azimuth relative to the measured localizer's location.

The flight inspection module is used for obtaining parameter data and position information from the RF signal acquisition and processing module, and then analyzing the flight inspection. Each sub module is also independent of each other. According to the allocated monitoring tasks, combining with the measured signal at each sampling point, and distance, azimuth and height information of the sample point between the runway entrance or the location of the monitored navigation equipment, every sub module can analyze its flight inspection results whether satisfies with the relevant requirements of "Flight Inspection Criterion for Civil Aviation Subgrade Navigation Equipment" or not.

\section{The Specific Steps of an On-Line Monitoring System}

At present, the navigation equipment on-line monitoring system usually based on handheld devices, they acquiring signal of navigation equipment point by point. The latest monitoring system based on moving vehicular can measure navigation equipment 's signal continuously, but they are often limited by the road, terrain, obstacles, etc. around the airport, the choice of measuring location is limited, and the measured signal in far field cannot be adopted because of their low measurement height and the obstacles occlusion. With the increasing of new airport and the surrounding buildings, the demand for flight inspection also greatly increase, but the professional personnel and plane for flight inspecting is very limited in our country currently, so that it is impossible for the airports in need to be flight inspected timely.

This paper provides an on-line monitoring and flight inspection system for navigation equipment, which is based on the UAV technology, its flight path and inspection methods are same as the traditional manned flight inspection, thus the system will not be impacted by the above factors. The system can measure signal of navigation equipment objectively, monitors and analysis signal effectively, its results are reliable.

The specific steps of the on-line monitoring and flight inspection method for navigation equipment are as follows:

Step 1: The central controller equips flight tasks, which including: the type of navigation equipment to be measured and its working frequency, the flight path of the UAV, and the navigation signal parameters.

Step 2: The airborne computer module sends flight commands to the actuator module according to the configured flight path, and the actuator module executes flight commands so that the UAV can flies along the configured flight path.

Step 3: After received satellite signals from GPS antenna and processed them, the GPS positioning module obtains the positioning information of UAV during its flight, and then sends the positioning information to the airborne computer module and the central controller.

Step 4: The measurement antenna receives the navigation equipment's signal and sends them to the RF acquisition and processing module.

Step 5: The RF acquisition and processing module acquires and processes the navigation equipment's signal, and then gets the parameters data of each sampling point, and each sample point's location information according to its positioning information, finally records and stores the parameters data and location information of current moment's sample point.

Step 6: The signal monitoring and analysis module monitors and analyzes the parameters data and location information from the RF acquisition and processing module, then sends the results to the central controller for graphical display.

Step 7: The flight inspection module obtains and flight inspects the parameters data and location information from the RF acquisition and processing module, and then sends the results to the central controller for graphical display.

Step 6 and Step 7 are mutual independent. We can only enable the signal monitoring and analysis module to execute Step 6 if our requirement is monitoring and analyzing the navigation signal. If our 
requirement is flight inspecting, then we can only enable the flight inspection module to execute Step 7. For the same reason, we can enable both the signal monitoring and analysis module and the flight inspection module is we want.

\section{Conclusion}

The paper proposes an on-line monitoring and flight inspection system for navigation equipment, which is based on unmanned aerial vehicle (UAV) technology. It is not impacted by the surrounding terrain, obstacles shielding, flight level and low visibility weather, measures navigation equipment's signal objectively and effectively, has a higher precision on monitoring and analyzing technology, gets a more reliable analysis results, so that can effectively reduce the impacts on the airport operation when measuring and flight inspecting the navigation equipment. With regards to the flight inspection department cannot flight inspecting in time for the demand airports because they are lack of professional personnel and plane, these airports can use the proposed on-line monitoring and flight inspection system to solve their problem, the airports can also use our system to deal with the periodic flight inspection. Employing our system for flight inspection not only can greatly reduce the time waiting for traditional manned flight inspection, save the costs of corresponding human and financial resources, but also can reduce the impacts on the airport operation caused by the traditional flight inspection.

\section{Acknowledgments}

This work was financially supported by the National Key R\&D Plan (2017YFB0503402). The authors would like to thank the anonymous reviewers and editors for their helpful comments and suggestions.

\section{References}

[1]. Sun Bo, Wang Yong. Navigation Device Tester based on ARM and $\mu \mathrm{C} / \mathrm{OS}-\mathrm{II}$, Instrument Technology and Sensors, 2011, vol. 4, pp: 23-24.

[2]. Ma Ertao, Li Jianhai, Liu Baohua, Wang Ping. Airborne short-range AIDS testing instrument design and implementation, Telecommunications Technology, 2010, vol. 50(3), pp: 69-72.

[3]. Jin K C, Sang H P, Cho D J, et al. Correction error generation algorithm for differential positioning performance analysis of navigation equipment[C], International Conference on Control, Automation and Systems. IEEE, 2008:1099-1103.

[4]. Johnson W S, Heller R A, Yang J N. Flight inspection data and crack initiation times [J]. 1977.

[5]. Reinagel F G, Johnson A B. Computerized flight inspection system: US, US 4792904 A [P]. 1988.

[6]. Kim E, Walter T, Powell J D. Unaugmented GPS-Based Flight Inspection System[J]. Aerospace \& Electronic Systems IEEE Transactions on, 2010, 46(2), pp: 717-724.

[7]. Chen Jian, Zheng Shiming, Liu Jia. Integrated navigation equipment ground testing system design and implementation, Electronic Design Engineer, 2011, vol. 19(10), pp: 110-113.

[8]. Li Gang, Wei Haitao, Sun Shuliang. Analysis of time delay measurement technology of navigation equipment, Radio Engineering, 2011, vol. 41(12), pp: 32-35. 